\title{
Hospital-Based Clinicians' Use of Technology for Patient Care-Related Communication: A National Survey
}

\author{
Kevin J. O'Leary, MD, MS ${ }^{1 *}$, David M. Liebovitz, MD², Robert C. Wu, MD, MSc ${ }^{3}$, Ksheeraja Ravi', Claire A. Knoten, PhD', \\ Mengxin Sun, MPP', Ashley M. Walker, MS ${ }^{5}$, Madhu C. Reddy, PhD ${ }^{5}$
}

\begin{abstract}
${ }^{1}$ Division of Hospital Medicine, Northwestern University Feinberg School of Medicine, Chicago, Illinois; ${ }^{2}$ Division of General Internal Medicine, University of Chicago, Chicago, Illinois; ' ${ }^{3}$ Department of Medicine, University of Toronto, Toronto, Canada; ${ }^{2}$ Northwestern University Feinberg School of Medicine, Chicago, Illinois; ${ }^{5}$ Department of Communication Studies, School of Communication, Northwestern University, Chicago, Illinois.
\end{abstract}

OBJECTIVE: To characterize current use of communication technologies, including standard text messaging and secure mobile messaging applications, for patient care-related (PCR) communication.

METHODS: We used a Society of Hospital Medicine database to conduct a national cross-sectional survey of hospital-based clinicians.

RESULTS: We analyzed data from 620 survey respondents (adjusted response rate, $11.0 \%$ ). Pagers were provided by hospitals to 495 (79.8\%) of these clinicians, and 304 (49\%) of the 620 reported they received PCR messages most commonly by pager. Use of standard text messaging for PCR communication was common, with 300 (52.9\%) of 567 clinicians reporting receipt of standard text messages once or more per day. Overall, 21.5\% (122/567) of respondents received standard text messages that included individually identifiable information, $41.3 \%$ (234/567) received messag- es that included some identifiable information (eg, patient initials), and $21.0 \%$ (119/567) received messages for urgent clinical issues at least once per day. About one-fourth of respondents $(26.6 \%, 146 / 549)$ reported their organization had implemented a secure messaging application that some clinicians were using, whereas few $(7.3 \%, 40 / 549)$ reported their organization had implemented an application that most clinicians were using.

DISCUSSION: Pagers remain the technology most commonly used by hospital-based clinicians, but a majority also use standard text messaging for PCR communication, and relatively few hospitals have fully implemented secure mobile messaging applications.

CONCLUSION: The wide range of technologies used suggests an evolution of methods to support communication among healthcare professionals. Journal of Hospital Medicine 2017;12:530-535. (C) 2017 Society of Hospital Medicine
Communication among healthcare professionals is essential for high-quality patient care. However, communication is difficult in hospitals because of heavy workloads, rapidly evolving plans of care, and geographic dispersion of team members. When hospital-based professionals are not in the same place at the same time, they rely on technology to communicate. Pagers have historically been used to support communication in hospitals, but are limited in their capabilities. Several recent small studies have shown that some physicians have started using standard text messaging on smartphones for patient care-related (PCR) messages. ${ }^{1-3}$ Although potentially enhancing clinician efficiency, use of standard text messaging for PCR messages raises concern about security risks related to transmission of protected health information. Addressing these concerns are emerging secure mobile messaging applications designed for PCR

\footnotetext{
*Address for correspondence and reprint requests: Kevin J. O'Leary, MD, MS, Division of Hospital Medicine, 211 E Ontario St, Suite 700, Chicago, IL 60611; Telephone: 312-926-5984; Fax: 312-926-4588; E-mail: keoleary@ nm.org

Additional Supporting Information may be found in the online version of this article.

Received: October 26, 2016; Revised: December 21, 2016; Accepted: January 1, 2017
}

2017 Society of Hospital Medicine DOI 10.12788/jhm.2767 communication. Although recent studies suggest these applications are well received by users, the adoption rate is largely unknown. ${ }^{4,5}$

We conducted a study to see if there was a shift in use of hospital-based communication technologies under way. We surveyed a national sample of hospital-based clinicians to characterize current use of communication technologies, assess potential risks and perceptions related to use of standard text messaging for PCR messages, and characterize the adoption of secure mobile messaging applications designed for PCR communication.

\section{METHODS}

\section{Study Design}

The study was a cross-sectional survey of hospitalistsphysicians and advanced practice providers whose primary professional focus is care of hospitalized patients. We studied hospitalists because of their role in coordinating care for complex medical patients and because prior studies identified communication as a major component of their work. ${ }^{6,7}$ The Northwestern University Institutional Review Board deemed this study exempt.

\section{Survey Instrument}

Four investigators (Drs. O'Leary, Liebovitz, Wu, and Reddy) with expertise in interprofessional communication and 
information technology created a draft survey based in part on results of prior studies assessing clinicians' use of smartphones and standard text messaging for PCR communication. ${ }^{1,3}$ In the first section of the survey, we asked respondents which technologies were provided by their organization and which technologies they used for PCR communication. In the second section, we asked respondents about their use and perceptions of standard text messaging for PCR communication. In the third section, we asked about implementation and adoption of secure mobile messaging applications at their hospital. In the fourth and final section, we asked for demographic information.

We randomly selected 8 attendees of the 2015 Midwest Hospital Medicine Conference and invited them to participate in a focus group that would review a paper version of the draft survey and recommend revisions. Using the group's feedback, we revised the ordinal response scale for questions related to standard text messaging and made other minor edits. We then created an Internet-based version of the survey and pilot-tested it with 8 hospitalists from 4 diverse hospitalist groups within the Northwestern Medicine Health System. We made additional minor edits based on pilot-test feedback.

\section{Sampling Strategy}

We used the largest hospitalist database maintained by the Society of Hospital Medicine (SHM). This database includes information on more than 28,000 individuals, representing SHM members and nonmembers who had participated in organizational events. In addition to clinically active hospitalists, the database includes non-hospitalists and clinically inactive hospitalists. We used this database to try to capture the largest possible number of potentially eligible hospitalists.

\section{Survey Administration}

We administered the survey in collaboration with SHM staff. E-mails that included a link to the survey on the Survey Monkey website were sent by SHM staff to individuals within the database. These e-mails were sent through Real Magnet, an e-mail marketing platform ${ }^{8}$ that allowed the SHM staff to determine the number of individuals who received and opened the e-mail and the number who clicked on the survey link. To try to promote participation, we offered respondents the chance to enter a lottery to win one of four $\$ 50$ gift certificates. The initial e-mail was sent in April 2016, a reminder in May 2016, and a final reminder in July 2016.

\section{Data Analysis}

We calculated descriptive statistics of participants' demographic characteristics. We estimated nonresponse bias by comparing demographic characteristics across waves of respondents using analysis of variance, $t$ tests, and $\chi^{2}$ tests. This method is based on the finding that characteristics of late respondents often resemble those of nonrespondents. ${ }^{9}$ We collapsed response categories for communication technologies to simplify interpretation. For example, numeric pagers, alphanumeric pagers, and 2-way pagers were collapsed into a pagers category. We used $t$ tests and $\chi^{2}$ tests to assess for associations between receipt of standard text messages for PCR communication and respondents' age, sex, race, professional type, hospital size, practice location, and hospital teaching status. Similarly, we used $t$ tests and $\chi^{2}$ tests to explore associations between implementation of secure mobile messaging application and respondents' age, sex, race, professional type, hospital size, practice location, and hospital teaching status. All statistical analyses were performed with Stata Release 11.2 (StataCorp).

\section{RESULTS}

\section{Participant Characteristics}

Overall, the survey link was sent to 28,870 e-mail addresses. Addresses for which e-mails were undeliverable or for which the e-mail was never opened were excluded, yielding a total of 5,786 eligible respondents in the sample. After rejecting 42 clinically inactive individuals, 70 individuals who responded to only the initial item, and 27 duplicates, a total of 620 participant surveys were included in the final analysis. The adjusted response rate was $11.0 \%$.

As shown in Table 1, mean (SD) respondent age was 42.9 (10.0) years, nearly half of the respondents were female, nearly a third were of nonwhite race, an overwhelming majority were physicians, and workplaces were in a variety of hospital settings. The sample size used to calculate demographic characteristics varied from 538 to 549 because of missing data for these items. We found no significant differences in demographic characteristics of respondents across the 3 survey waves, suggesting a lack of survey response bias (Supplemental Table).

\section{Provision and Use of Communication Technologies for PCR Communication}

Pagers were provided to the majority of respondents by their hospitals $(79.8 \%, 495 / 620)$. Other devices were provided much less frequently, with $21.0 \%$ (130/620) reporting their organization provided a smartphone, $20.2 \%(125 / 620)$ a mobile phone, and 4.4\% (27/620) a hands-free communication device. Organizations provided no device to $8.2 \%(51 / 620)$ of respondents and an "other" device to 5.5\% (34/620).

An overwhelming majority used multiple technologies to receive PCR communication, with $17.7 \%(110 / 620)$ of respondents indicating use of 2 technologies, $22.7 \%(141 / 620)$ use of 3 technologies, and $49.4 \%$ (306/620) use of more than 3 technologies. The distribution of the most common ways respondents received PCR communication is shown in Table 2. Pagers were the most common form of technology, with $49.0 \%(304 / 620)$ indicating this was the primary way they received PCR communication. Being called on a mobile phone provided by the organization was the second most common form of receiving PCR communication (11.0\%, $68 / 620)$, followed by standard text messaging $(9.5 \%, 59 / 620)$ and mobile secure messaging using an application approved by the organization $(9.0 \%, 56 / 620)$. 


\section{TABLE 1. Participant Characteristics}

\begin{tabular}{|c|c|}
\hline Characteristic, n (\%) & $\begin{array}{l}\text { Participants } \\
(\mathrm{n}=549)\end{array}$ \\
\hline Mean (SD) age, y & $42.9(10.0)$ \\
\hline Female sex & $265(48.3)$ \\
\hline \multicolumn{2}{|l|}{ Race } \\
\hline White & $380(69.2)$ \\
\hline Asian & $108(19.7)$ \\
\hline Black & $20(3.6)$ \\
\hline Hispanic & $14(2.6)$ \\
\hline Other & $27(4.9)$ \\
\hline \multicolumn{2}{|l|}{ Professional type } \\
\hline Physician & $482(87.8)$ \\
\hline Nurse practitioner or physician assistant & $67(12.2)$ \\
\hline \multicolumn{2}{|l|}{ Hospital size, number of beds ${ }^{\mathrm{a}}$} \\
\hline$<100$ & $45(8.4)$ \\
\hline $100-299$ & $154(28.6)$ \\
\hline $300-499$ & $147(27.3)$ \\
\hline$\geq 500$ & $192(35.7)$ \\
\hline \multicolumn{2}{|l|}{ Practice location } \\
\hline Northeast & $110(20.0)$ \\
\hline Midwest & $140(25.5)$ \\
\hline South & $157(28.6)$ \\
\hline West & $125(22.8)$ \\
\hline Other & $17(3.1)$ \\
\hline \multicolumn{2}{|l|}{ Teaching status } \\
\hline Major teaching hospital & $242(44.1)$ \\
\hline Teaching hospital & $196(35.7)$ \\
\hline Nonteaching hospital & $110(20.0)$ \\
\hline WiFi connection available in workplace & 535 (97.5) \\
\hline
\end{tabular}

\section{Participants' Experiences With Standard Text Messaging for PCR Communication}

Participants' experiences with standard text messaging for PCR communication are summarized in Table 3. Overall, $65.1 \%$ (369/567) of respondents reported receiving standard text messages for PCR communication at least once per week when on clinical duty, and 52.9\% (300/567) received standard text messages at least once per day.

Overall, 21.5\% (122/567) of respondents received standard text messages that included individually identifiable information at least once per day, and 41.3\% (234/567) re-
TABLE 2. Technologies Used to Receive Patient Care-Related Communication

\begin{tabular}{ll}
\hline $\begin{array}{l}\text { What is the most common way you receive } \\
\text { patient care-related messages? (descending order } \\
\text { of frequency), } n(\%)\end{array}$ & $\begin{array}{c}\text { Participants } \\
(\mathrm{n}=620)\end{array}$ \\
\hline Pager & $304(49.0)$ \\
\hline Call to mobile phone provided by hospital & $68(11.0)$ \\
\hline Text messaging using standard text messaging & $59(9.5)$ \\
\hline Text messaging application approved by organization & $56(9.0)$ \\
\hline Call to smartphone & $53(8.6)$ \\
\hline Messaging within electronic health record & $32(5.2)$ \\
\hline E-mail to professional account & $20(3.2)$ \\
\hline Hands-free communication device & $6(1.0)$ \\
\hline E-mail to personal account & $3(0.5)$ \\
\hline Other & $19(3.1)$ \\
\hline
\end{tabular}

ceived messages that included some identifiable information (eg, patient initials, room number) at least once per day. About one-fifth of respondents $(21.0 \%, 119 / 567)$ indicated receiving standard text messages for urgent clinical issues at least once per day. Receipt of standard text messages for a patient for whom the respondent was no longer providing care, delays in receipt of messages, messages missed because smartphones were set to vibrate, and receipt of messages when not on clinical duty occurred, but less frequently. We found no significant associations between receipt of PCR standard text messages once or more per day and respondents' age, sex, race, professional type, hospital size, or hospital teaching status. A higher percentage of respondents in the South $(63.2 \%, 96 / 152)$ and West $(57.9 \%, 70 / 121)$ reported receipt of at least 1 PCR standard text message per day, compared with respondents in the Northeast $(51.9 \%, 54 / 104)$, Midwest $(45.2 \%, 61 / 135)$, and other $(25.0 \%, 4 / 16)(P=0.003)$.

Senders of PCR standard text messages. Of respondents who received standard text messages for PCR communication at least once per week, a majority reported receiving messages from physicians in the same specialty $(88.6 \%, 327 / 369)$ and from physicians in other specialties $(71.3 \%, 263 / 369)$. A minority of respondents reported receiving messages from nurses $(35.0 \%, 129 / 369)$, social workers $(30.6 \%, 113 / 369)$, and pharmacists $(27.9 \%, 103 / 369)$.

Perceptions among users. Of respondents who received standard text messages for PCR communication at least once per week, an overwhelming majority agreed or strongly agreed that use of standard text messaging allowed them to provide better care $(81.7 \%, 295 / 361)$ and made them more efficient $(87.3 \%, 315 / 361)$. A majority also agreed or strongly agreed that standard text messaging posed a risk to the privacy and confidentiality of patient information (56.4\%, 203/360), and nearly a third indicated that standard text messaging 
TABLE 3. Participants' Experiences With Standard Text Messaging for Patient Care-Related Communication

\begin{tabular}{|c|c|c|c|c|}
\hline \multirow[b]{2}{*}{$\begin{array}{l}\text { How often do you ... } \\
n(\%)\end{array}$} & \multicolumn{4}{|c|}{ Frequency $(n=567)$} \\
\hline & $\begin{array}{c}\text { Never/Less than } \\
\text { once a week }\end{array}$ & $\begin{array}{c}\text { Between once a } \\
\text { week } \\
\text { and once a day }\end{array}$ & $\begin{array}{l}\text { 1-3 times } \\
\text { a day }\end{array}$ & $\begin{array}{c}4 \text { or more times } \\
\text { a day }\end{array}$ \\
\hline Receive patient care-related text messages & $198(34.9)$ & $69(12.2)$ & $105(18.5)$ & $195(34.4)$ \\
\hline Receive text messages that include individually identifiable health information & $392(69.1)$ & $53(9.4)$ & $53(9.4)$ & $69(12.2)$ \\
\hline Receive text messages that include some identifiable information & $236(41.6)$ & $97(17.1)$ & $96(16.9)$ & $138(24.3)$ \\
\hline Receive text messages that include a patient care-related image & $505(89.1)$ & $35(6.2)$ & $19(3.4)$ & $8(1.4)$ \\
\hline Receive patient care-related text messages for urgent clinical issues & $341(60.1)$ & $107(18.9)$ & $69(12.2)$ & $50(8.8)$ \\
\hline Receive text messages regarding a patient for whom you no longer provide care & $512(90.3)$ & $45(7.9)$ & $6(1.1)$ & $4(0.7)$ \\
\hline Experience delays in receiving messages related to patient care & $503(88.7)$ & $46(8.1)$ & $13(2.3)$ & $5(0.8)$ \\
\hline Experience difficulty receiving patient care-related messages because of battery running out & $535(94.4)$ & $27(4.8)$ & $5(0.9)$ & $0(0)$ \\
\hline Miss a patient care-related message because device is set to vibrate & $462(81.5)$ & $75(13.2)$ & $27(4.8)$ & $3(0.5)$ \\
\hline Receive patient care-related text messages when not on clinical duty & $439(77.4)$ & $107(18.9)$ & $18(3.2)$ & $3(0.5)$ \\
\hline
\end{tabular}

posed a risk to the timely receipt of messages by the correct individual $(27.6 \%, 100 / 362)$. Overall, a large majority agreed or strongly agreed that the benefits of using standard text messaging for PCR communication outweighed the risks $(85.0 \%, 306 / 360)$.

\section{Adoption of Organization-Approved Secure Mobile Messaging Applications}

Participants' reported adoption of organization-approved secure mobile messaging applications is shown in the Figure. About one-fourth $(26.6 \%, 146 / 549)$ of respondents reported that their organization had implemented a secure messaging application and that some clinicians were using it, whereas relatively few $(7.3 \%, 40 / 549)$ reported that their organiza-

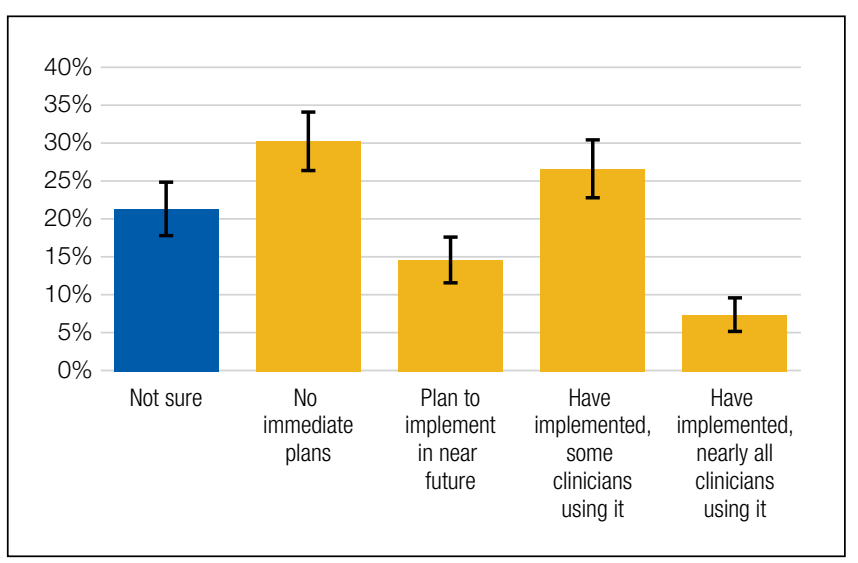

FIG. Reported adoption of secure mobile messaging applications for patient care-related communication.

NOTE: $\mathrm{N}=549$. Error bars indicate 95\% confidence intervals for each calculated proportion. tion had implemented an application that was being used by most clinicians. A substantial portion of respondents (21.3\%, $117 / 549)$ were not sure whether their organization was planning to implement a secure mobile messaging application for PCR communication. We found no significant associations between partial or nearly full implementation of a secure mobile messaging application and respondents' age, sex, race, professional type, hospital size, or practice location. A lower percentage of respondents in major teaching hospitals $(28.0 \%, 67 / 239)$ reported partial or nearly full implementation of a secure mobile messaging application, compared with respondents from teaching hospitals $(39.6 \%, 74 / 187)$ and nonteaching hospitals $(39.2 \%, 40 / 102)(P=0.02)$.

\section{DISCUSSION}

We found that pagers were the technology most commonly used by hospital-based clinicians, but also that a majority have used standard text messaging for PCR communication, and that relatively few hospitals had fully implemented secure mobile messaging applications. Our findings reveal a wide range of technology use and suggest an evolution to support communication among healthcare professionals.

The persistence of pagers as the technology most commonly provided by hospitals and used by clinicians for communication is noteworthy in that pagers are limited in their capabilities, typically not allowing a response to the message sender or the ability to forward a message, and often not allowing the ability to send messages to multiple recipients. The continued heavy use of pagers may be explained by their relatively low cost, especially compared with investment in new technologies, and reliable receipt of messages, even in areas with no cell phone service or WiFi signal. Furthermore, hospitals' providing pagers allows for oversight, 
directory creation, and the potential for integration into other information systems. In 2 recent studies, inpatient paging communication was evaluated in depth. Carlile et al. ${ }^{10}$ found that the majority of pages requested a response, requiring an interruption in physician workflow to initiate a callback. Kummerow Broman et al. ${ }^{11}$ similarly found that a majority of pages requested a callback; they also found a high volume of nonurgent messages. With pager use, a high volume of messages, many of which require a response but are nonurgent, makes for a highly interruptive workflow.

That more than half of our hospital-based clinicians received standard text messages for PCR communication once or more per day is consistent with other, smaller studies. Kuhlmann et al. ${ }^{1}$ surveyed 97 pediatric hospitalists and found that a majority sent and received work-related text messages. Prochaska et al. ${ }^{2}$ surveyed 131 residents and found that standard text messaging was the communication method preferred by the majority of residents. Similar to these studies, our study found that receipt of standard text messages that included protected health information was fairly common. However, we identified additional risks related to standard text messaging. One-fifth of our respondents received standard text messages for urgent clinical issues once or more per day, and many respondents reported occasional receipt of messages regarding a patient for whom they were no longer providing care and receipt of messages when not on clinical duty. The usual inability to automate forwarding of standard text messages to another clinician creates the potential for clinically important messages to be delayed or missed. These risks have not been reported in the literature, and we think healthcare systems may not be fully aware of them. Our findings suggest that many clinicians have migrated from pagers to standard text messaging for the enhanced efficiency, and they perceive that the benefit of improved efficiency outweighs the risks to protected health information and the delay in receipt of clinically important messages by the correct individual.

Secure mobile messaging applications seem to address the limitations of both pagers and standard text messaging. Secure mobile messaging applications typically allow message response, message forwarding, multiple recipients, directory creation, the potential to create escalation schemes for nonresponse, and integration with other information systems, including electronic health records. Although several hospitals have developed their own systems, ${ }^{4,12,13}$ most hospitals likely will purchase a vendor-based system. We found that a minority of hospitals had implemented a secure messaging application, and even fewer had most of their clinicians using it. Although little research has been conducted on these applications, studies suggest they are well received by users. ${ }^{4,5}$ Given that paging communication studies have found a large portion of pages are sent by nurses and other non-physician team members, secure mobile messaging applications should allow for direct message exchange with all professionals caring for a patient. ${ }^{10,11}$ Furthermore, hospitals will need to ensure adequate cell phone and $\mathrm{WiFi}$ signal strength throughout their facilities to ensure reliable and timely delivery of messages.

Our study had several limitations. We used a large database to conduct a national survey but had a low response rate and some drop-off of responses within surveys. Our sample reflected respondent diversity, and our analyses of demographic characteristics found no significant differences across survey response waves. Unfortunately, we did not have nonrespondents' characteristics and therefore could not compare them with respondents'. It is possible that nonrespondents may have had different practices related to use of communication technology, especially in light of the fact that the survey was conducted by e-mail. However, given our finding that use of standard text messaging was comparable to that in other studies, ${ }^{1,2}$ and given the similarity of respondents' characteristics across response waves, our findings likely were not affected by nonresponse bias. ${ }^{9}$ Last, we used a survey that had not been validated. However, this survey was created by experts in interprofessional collaboration and information technology, was informed by prior studies, and was iteratively refined during pretesting and pilot testing.

\section{CONCLUSION}

Pagers remain the technology most commonly used by hospital-based clinicians, but a majority also use standard text messaging for PCR communication, and relatively few hospitals have fully implemented secure mobile messaging applications. The wide range of technologies used suggests an evolution of methods to support communication among healthcare professionals. An optimized system will improve communication efficiency while ensuring the security of their patients' information and the timely receipt of that information by the intended clinician.

\section{Acknowledgment}

The authors thank the Society of Hospital Medicine and the society staff who helped administer the survey, especially Mr. Ethan Gray.

Disclosure: Nothing to report.

\section{References}

1. Kuhlmann S, Ahlers-Schmidt CR, Steinberger E. TXT@WORK: pediatric hospitalists and text messaging. Telemed J E Health. 2014;20(7):647-652.

2. Prochaska MT, Bird AN, Chadaga A, Arora VM. Resident use of text messaging for patient care: ease of use or breach of privacy? JMIR Med Inform. 2015;3(4):e37.

3. Tran K, Morra D, Lo V, Quan SD, Abrams H, Wu RC. Medical students and personal smartphones in the clinical environment: the impact on confidentiality of personal health information and professionalism. J Med Internet Res. 2014;16(5):e132.

4. Patel N, Siegler JE, Stromberg N, Ravitz N, Hanson CW. Perfect storm of inpatient communication needs and an innovative solution utilizing smartphones and secured messaging. Appl Clin Inform. 2016;7(3):777-789.

5. Przybylo JA, Wang A, Loftus P, Evans KH, Chu I, Shieh L. Smarter hospital communication: secure smartphone text messaging improves provider satisfaction and perception of efficacy, workflow. J Hosp Med. 2014;9(9):573-578.

6. O'Leary KJ, Liebovitz DM, Baker DW. How hospitalists spend their time: insights on efficiency and safety. J Hosp Med. 2006;1(2):88-93.

7. Tipping MD, Forth VE, O'Leary KJ, et al. Where did the day go?- - a time-motion study of hospitalists. J Hosp Med. 2010;5(6):323-328.

8. Real Magnet. http://www.realmagnet.com. Accessed December 20, 2016.

9. Armstrong JS, Overton T. Estimating nonresponse bias in mail surveys. J Mark Res. 1977;14(3):396-402. 
10. Carlile N, Rhatigan JJ, Bates DW. Why do we still page each other? Examining the frequency, types and senders of pages in academic medical services. BMJ Qual Saf. 2017;26(1):24-29.

11. Kummerow Broman K, Kensinger C, Phillips C, et al. Characterizing the clamor: an in-depth analysis of inpatient paging communication. Acad Med. 2016;91(7):1015-1021.
12. Dalal AK, Schnipper J, Massaro A, et al. A web-based and mobile patient-centered "microblog" messaging platform to improve care team communication in acute care. J Am Med Inform Assoc. 2017;24(e1):e178-e184.

13. Wu R, Lo V, Morra D, et al. A smartphone-enabled communication system to improve hospital communication: usage and perceptions of medical trainees and nurses on general internal medicine wards. J Hosp Med. 2015;10(2):83-89. 